\title{
Metodología de trabajo en grupo para aprender programación de computadores
}

\section{A teamwork's methodology for learning computer programming}

\begin{abstract}
Omar Iván Trejos Buriticá
Universidad Tecnológica de Pereira

La Julita, Colombia.

\section{Resumen}

Objetivo: Brindar elementos de juicio al docente para que concretice un modelo de trabajo en grupo efectivo en la enseñanza-aprendizaje de la programación de computadores en Ingeniería. Método: Se adoptó una estrategia de trabajos en grupo durante 6 semestres a partir de tres modelos: modelo 4Q, modelo de talento académico y modelo de agrupamiento aleatorio. Resultados: Los resultados permiten entender las interacciones de cada grupo y su dinámica interna. Conclusión: Se concluyó que es posible escoger un modelo de conformación de grupos de trabajo en programación de computadores para la Ingeniería si se cuenta con bases inspiradas en la investigación científica que a su vez se apoyen en las Ciencias de la Educación.
\end{abstract}

Palabras clave: Aprendizaje, ingeniería de sistemas, modelo 4Q, modelo aleatorio, programación de computadores, trabajo en grupo.

\section{Abstract}

Objective: To offer elements of judgment to the teacher so that it concretizes an effective group work model in the teaching-learning of the computer programming in Engineering. Method: A group work strategy was adopted for 6 semesters based on three models: model $4 \mathrm{Q}$, academic talent model and random grouping model. Results: The results allow to understand the interactions of each group and their internal dynamics. Conclusion: It was concluded that it is possible to choose a model for the formation of working groups in computer programming for Engineering if there are bases based on scientific research that in turn are supported by the Education Sciences.

Keywords: 4Q model, computer programming, learning, random model, Systems Engineering, teamwork.
ISSN: 0124-2121 E-ISSN: $2665-2420$

Open Access:

Editor:

Patricia Martínez Barrios Universidad Simón Bolivar

Correspondencia: Omar Trejos Buriticá omartrejos@utp.edu.co

Recibido: 03-03-18 Aceptado: $30-08-18$

Publicado: $12-12-18$ um.21.36.3002

Cómo citar este artículo (APA): Trejos, O. (2019). Metodología de trabajo en grupo para aprender programación de computadores. Educación y Humanismo, 21(36), 23-39.DOI: http://dx10.17081/eduhum.21.36.3002 


\section{Introducción}

En el proceso de formación de los estudiantes, una de las competencias más complejas y menos logradas es la de contribuir en su capacidad para formar parte de grupos de trabajo (teamworks) y de alcanzar objetivos conjuntos a partir de la complementación de sus talentos y capacidades. Entrenar a los estudiantes en esta competencia permite que, a futuro, ya en su vida profesional, puedan participar de grupos interdisciplinarios que esa misma vida les exige. En concreto, así ocurre con los ingenieros de sistemas, quienes, con frecuencia, deben formar parte de grupos de trabajo institucionales conformados por profesionales de distintas áreas (contadores, economistas, administradores, ingenieros de otras áreas, matemáticos, etc.).

En este sentido, una de las quejas más frecuentes de los empresarios radica en que, a pesar de la alta capacidad técnica, tecnológica y metodológica de los ingenieros de sistema, su participación en grupos de trabajo es notoriamente débil, dado que la dinámica propia de estos grupos invita a pensar en actividades como debates, argumentaciones, foros, interacciones, coincidencias, discrepancias, y todo tipo de formas sociales de confrontación y complementación de ideas. A la luz de estas necesidades, los docente tenemos una responsabilidad superior a la que el conocimiento disciplinar establece, y es la de formar a nuestros estudiantes en estas lides, de manera que su salto a la vida profesional no solo consista en participar de las soluciones que la sociedad les exige desde su conocimiento científico, sino también un paso trascendental que les lleve a convertirse en personas que interactúen y participen en las dinámicas propias de la vida social, tanto en lo puramente profesional como en lo externo.

Desde este proyecto de investigación, se intenta aprovechar el paso de los estudiantes por la universidad para entrenarlos en estas competencias, de modo que, a partir de la experiencia recogida en tal sentido, este artículo se presenta como uno de sus productos. La pregunta de investigación que este artículo trata de responder es, entonces, la siguiente: ¿Cuál ha de ser el modelo más adecuado para la conformación de grupos, en el ámbito académico, de manera que el estudiante pueda desarrollar la competencia de formar parte activa, efectiva, eficiente y eficaz de un grupo o equipo de trabajo? Se espera aportar elementos de discusión bien sea para que se adopten en el aula de clases

en los procesos de formación en ingeniería, o para contar con nuevos elementos de juicio que nutran la discusión al respecto.

Más específicamente, a pesar de que, en los programas de Ingeniería, se acude a la conformación de grupos de trabajo como estrategia de trabajo académico, pocas veces se 
analiza la incorporación de esta estrategia desde la óptica de modelos definidos y estructurados que permitan inferir conclusiones 0 , por lo menos, aportar elementos para la discusión. Lo novedoso en este artículo es que no sólo resalta la adopción de la estrategia de trabajo en grupo como un camino para fortalecer el aprendizaje, sino que busca establecer criterios de conformación basados en modelos claramente definidos y, además, establece unos elementos de juicio para, a su vez, establecer parámetros comparativos en relación con la interacción de cada estudiante y su respectivo grupos a la luz de los modelos adoptados. Todo ello con la intención ya planteada de fortalecer el trabajo en grupo como parte de la preparación para la vida profesional.

Esta investigación se justificó, además, porque con mucha frecuencia, en los programas de ingeniería, se deben desarrollar tareas a partir de actividades grupales de los estudiantes.

Ahora bien, aunque la conformación aleatoria de grupos es posiblemente la técnica más utilizada, no siempre es la más acertada, debido a que el estudiante, especialmente de primeros semestres, queda a discreción de lo que la suerte le depare en lo que se refiere a los talentos y capacidades de sus compañeros. Por tal razón este estudio se realizó con los estudiantes de la asignatura Programación I de $1^{0}$ semestre de Ingeniería de Sistemas y Computación de la Universidad Tecnológica de Pereira durante los semestres I 2014, II 2014, I 2015, II 2015, I 2016 y II 2016.

Cabe mencionar que para el desarrollo de este proyecto de investigación, y en especial de lo que corresponde al contenido de este artículo, se acudió a una literatura especializada y se han adoptado elementos de juicio de teorías de aprendizaje, modelos de pensamiento $y$, a partir también de la experiencia, se han incorporado modelos de agrupamiento de estudiantes. Por lo dicho, el objetivo de este artículo consiste en presentar los resultados obtenidos del proyecto asociado en el marco de una investigación que, teniendo elementos cuantitativos, fundamenta sus inferencias a partir de los elementos cualitativos que lo componen y que incluyen estudios de casos individuales y grupales a la luz de una temática específica de conocimiento disciplinar.

De acuerdo con lo anterior, ¿es posible fortalecer el trabajo en grupo, capitalizar sus bondades para beneficiar el logro de objetivos individuales de aprendizaje de los estudiantes de programación de computadores en Ingeniería de Sistemas y, además, propiciar las actividades propias de la dinámica de los grupos como un camino para fomentar la competencia profesional de participación del futuro ingeniero en grupos y equipos de trabajo? La respuesta, en parte, es el este artículo, que desde la experiencia, los resultados y las conclusiones aporta elementos de juicio para la discusión respectiva. 


\section{Marco teórico}

El aprendizaje es el acto a través del cual se adquiere, se modifica o se refuerza el conocimiento existente, los comportamientos, las habilidades, los valores o las preferencias, de manera que pueden conducir a un cambio en la sintetización de la información, a una profundización del mismo conocimiento, y a una actitud o comportamiento relativos a un tipo y rango de experiencias (Attard, Di Ioio, \& Geven, 2010). La habilidad de aprender es propia de los humanos, animales, plantas y aun de algunas máquinas. A través del tiempo, el progreso tiende a seguir una determinada curva de aprendizaje. Y el aprendizaje no sucede de una vez: se construye y adquiere forma a partir del conocimiento previo (Ausubel, 1986). En consecuencia, el aprendizaje puede ser visto como un proceso, más que como una colección de hechos y procedimientos que produce cambios de carácter permanente en los organismos. El aprendizaje humano ocurre como parte de los procesos educativos, el desarrollo personal, la incorporación a la escuela o el entrenamiento; está orientado, de otro lado, por determinados objetivos y se soporta en la motivación (Bruner, 2009). El aprendizaje puede ocurrir como resultado de los condicionamientos clásicos o habituales, que pueden ser observados en muchas especies animales o como el resultado de actividades más complejas como los juegos, lo cual puede ser observado solo en animales inteligentes (Kapp, 2012). El estudio de la forma como sucede el aprendizaje es parte de la sicología educativa, la neurosicología, la teoría del aprendizaje y la pedagogía.

El aprendizaje puede ocurrir en forma consciente 0 inconsciente. Incluso, existe evidencia de que el comportamiento humano se afecta por el aprendizaje prenatal, lo cual se ha observado a lo largo de las 32 semanas de gestación, estableciéndose que el sistema nervioso central está suficientemente desarrollado y motivado por el aprendizaje y la memoria en estas etapas tempranas de la formación. En otro sentido, diferentes teóricos han reconocido el juego como la primera forma de aprendizaje. Según Piaget (2001), los niños aprenden las reglas y aprenden a interactuar jugando. Otros científicos piensan que el juego es esencial para el desarrollo de los niños, debido a que les permite encontrar significado a su entorno, a sus interacciones y a su relación con ellos. En el mundo moderno, la labor del docente universitario va mucho más allá de impartir un conocimiento puramente magistral (Barriga Arceo \& Hernandez-Rojas, 2002).

Existe una gran cantidad de estrategias que permiten a un estudiante recorrer un camino más expedito para adquirir conocimiento y estas estrategias incluyen, entre muchas otras, el trabajo en grupo. Unirse con otras personas para alcanzar un objetivo de aprendizaje representa cinco ventajas que son de una alta importancia para el estudiante de los tiempos modernos (Dooly, 2008): a) interactuar con otras personas y establecer relaciones de cercanía alrededor de un mismo tema, b) confrontar sus ideas y soportarlas en argumentos convincentes sobre una base lógica práctica, c) escuchar ideas diferentes a 
las propias, lo que forma el pensamiento crítico no sólo frente a lo que piensan los demás sino también frente a lo que el mismo estudiante piensa sobre una situación específica, d) unir esfuerzos, habilidades, destrezas y competencias para lograr, conjuntamente, un objetivo de aprendizaje y e) aprender de manera colectiva, beneficiando tanto al grupo como a sí mismo. Los grupos de trabajo constituyen, así, una de las estrategias más comúnmente adoptadas por los docentes universitarios para el desarrollo de sus actividades pedagógicas de cara al logro de sus objetivos (Adams, 2013).

El término teamwork (grupo de trabajo) se refiere a una serie de procesos comportamentales y como un estado que surge cuando un grupo de personas quieren alcanzar un objetivo común. En el mundo de la medicina, por ejemplo, el concepto de teamwork involucra dos o más profesionales con habilidades y conocimientos complementarios, que comparten objetivos comunes y que se han ejercitado en esfuerzos físicos y mentales conjuntos para asesorar, planear o evaluar los cuidados de un paciente.

Por su parte, en el mundo de los negocios, las técnicas conjuntas de acompañamiento pueden ser usadas para proveer medidas financieras beneficiosas (Terenzini, Cabrera, Colbeck, Parente \& Bjorklund, 2001). En Ingeniería, el trabajo en grupo se hace altamente necesario porque el ingeniero debe interactuar con profesionales de otras áreas para realizar su labor. El ingeniero de sistemas es posiblemente el profesional de la ingeniería que más requiere de esa interacción con otros profesionales, con otros conocimientos y con otras habilidades, dado que si su objetivo profesional gira alrededor de la información, son otros profesionales los que pueden ilustrarle acerca del uso de esta según el campo en el cual se desempeñe (Blanchard, 2000). A nivel empresarial, la relación que se establece cuando un ingeniero de sistemas interactúa con contadores, economistas, ingenieros de otras áreas, administradores, etc., le proveen de diferentes miradas que enriquecen su propio perfil y favorecen su presencia en un determinada empresa que, muchas veces, es catapultada por el aprovechamiento de las nuevas tecnologías de la información y la comunicación (Trejos-Buriticá, 2013).

Pasando a otro aspecto de nuestro problema de estudio, el primer modelo que se ha adoptado para esta investigación se conoce como Modelo $4 \mathrm{Q}$ de Preferencias de Pensamiento, y ayuda a los investigadores, a los equipos, a los grupos de trabajo y a las empresas a alcanzar los mejores beneficios de las personas que dirigen. Según este modelo, mientras diferentes tareas requieren procesos mentales disímiles, y las personas tienen formas de pensar distintas, las organizaciones pueden obtener unos resultados mejores si conocen el espectro posible de pensamientos y enfoques de los distintos seres humanos (Lumsdaine \& Lumsdaine, 2005). Los seres humanos tienen preferencias de pensamiento que en algunos son muy fuertes $y$, en otros, más moderadas. Estas preferencias desarrollan determinados niveles de dominación y no siempre se es consciente de estas, al punto que muchas veces dos personas pueden tener puntos de 
vista que parecieran distintos y coincidir en sus preferencias de pensamiento (Herrmann, 2004). El modelo de preferencias de pensamiento llamado $4 Q$ indica que cada uno tiene acceso a cuatro formas de pensamiento y que una de ellas domina a los demás cuadrantes cuando sea requerido, cuando necesite adaptarse o cuando necesite sacar ventaja en determinada situación tendiente a mejorar el rendimiento y alcanzar mejores resultados. La Figura 1 presenta un diagrama del modelo 4Q referenciado.

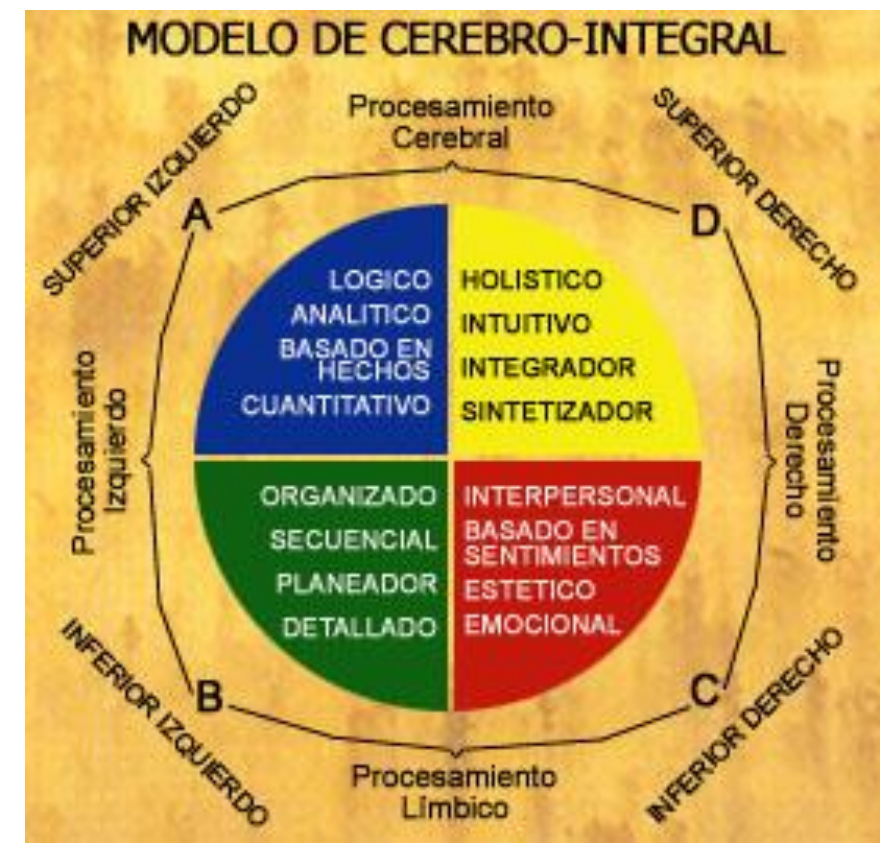

Figura 1. Modelo $4 \mathrm{Q}$ de preferencias de pensamiento Fuente: William Herrmann (2004).

De acuerdo con este modelo, el cuadrante A corresponde al pensamiento lógico analítico, que es el que permite que las personas con este pensamiento preferente sean las más indicadas para recoger datos y analizarlos, comprender cómo funcionan las cosas, evaluar ideas basadas en hechos y también para el razonamiento lógico. En el cuadrante B se ubica el pensamiento secuencial, en el que se ubican aquellos que suelen ser muy hábiles para seguir linealmente un conjunto de instrucciones, para realizar trabajos claramente orientados, para encontrar solución a un problema desarrollándola paso a paso, así como para organizar e implementar una determinada solución. El cuadrante C tiene relación con el pensamiento interpersonal, es el cuadrante social. Las personas ubicadas en él desarrollan una mayor capacidad para escuchar y expresar sus ideas, para buscar y encontrar lo que pareciera importante para las demás, para analizar las características de grupos de interacción $y$, en algunos casos, para liderarlos. El 40 cuadrante es el cuadrante D o imaginativo. En este cuadrante se ubican las personas capaces de observar el paisaje y no se detienen a mirar solamente un árbol, que pueden tomar la iniciativa y que asumen retos por ellos mismos. Son mucho más visuales y suelen 
pensar con un alto componente metafórico, lo que les permite poseer una capacidad de resolver creativamente los problemas, además de tener un pensamiento que se distingue a largo plazo por sus características.

El segundo modelo que se ha adoptado en esta investigación puede llamarse Modelo de Talento Académico. En este la clasificación de estudiantes se basa en la experiencia tanto del autor de este artículo como de otros docentes e investigadores. Los estudiantes pueden ubicarse en una de tres categorías: estudiantes Altamente Talentosos (AT), estudiantes Medianamente Talentosos (MT) y estudiantes Bajamente Talentosos (BT).

Los estudiantes Altamente Talentosos (AT) tienen un conocimiento disciplinar notoriamente sólido, tanto sus habilidades como sus competencias están en rima con dicho conocimiento disciplinar y tienen la capacidad de aprovechar cualquier oportunidad para aplicarlo. Por su parte, los estudiantes Bajamente Talentosos (BT) son aquellos que cumplen una de las siguientes tres condiciones: a) no poseen un conocimiento disciplinar en ningún nivel, b) tanto sus habilidades como sus competencias parecieran no estar en rima con lo que la asignatura y el programa de formación profesional requiere y c) normalmente no manifiestan un interés pronunciado para involucrarse en aquellas situaciones donde dicho conocimiento se puede aplicar. Por último, los estudiantes Medianamente Talentosos (MT) son los que a pesar de tener un conocimiento disciplinar débil y difuso, parecen tener habilidades y competencias que coinciden con los requerimientos del programa de formación, pero aún no han aflorado en ellos y, por ende, ni siquiera ellos mismos, muchas veces, lo saben; además, participan tímidamente en las situaciones en las cuales se requiere aplicar el conocimiento aunque muestran gran interés en estar allí.

El tercer modelo que se ha adoptado en esta investigación corresponde al modelo aleatorio de agrupamiento. En este, el estudiante hace grupo con cualquier persona sin que medie ningún modelo específico. Este modelo de agrupamiento es completamente aleatorio cuando se incorpora como estrategia en las asignaturas de los primeros semestres y, de manera muy especial, en el $1^{0}$ semestre de cualquier programa de formación profesional. $Y$ es el modelo al que se enfrenta el estudiante en su vida profesional, salvo los casos en los cuales tiene la potestad de escoger su equipo de trabajo. La enseñanza y el aprendizaje de la programación de computadores se ha convertido en una alta necesidad que supone un reto en dos sentidos: al estudiante le impone el reto de aprender a programar, es decir, de apropiar, asimilar, aplicar y evaluar las técnicas, teorías, tecnologías, modelos y metodologías que subyacen en un determinado paradigma de programación (Van Roy, 2008). Por su parte, el docente debe adoptar estrategias que le posibiliten al estudiante alcanzar sus logros de aprendizaje (Trejos-Buriticá, 2012), es decir, aprender a programar por caminos que no solo fomenten la apropiación del conocimiento disciplinar sino que, además, lo formen para incorporarse 
a la vida profesional de la manera más efectiva (Prince, 2004).

\section{Método}

Tal como se expuso, este estudio se realizó con los estudiantes de la asignatura Programación I del $1^{0}$ semestre de Ingeniería de Sistemas y Computación de la Universidad Tecnológica de Pereira durante los semestres I 2014, II 2014, I 2015, II 2015, I 2016 y II 2016. Para obtener unos resultados que, de manera comparativa, permitieran inferir conclusiones próximas a la realidad, al inicio de cada curso se tomaron las dos primeras semanas para analizar a los estudiantes de los cuales poco se sabía (excepto sus datos personales), dado que eran estudiantes de primer semestre. A través de observación, pruebas escritas, modelos esquemáticos de caracterización de estudiantes y entrevistas personalizadas, se reconoció a los estudiantes que, de manera más marcada, se ubicaran claramente dentro de los cuadrantes del modelo 4Q o del modelo de talento académico. Se intentó identificar al menos 2 estudiantes por cada categoría, esto es, 2 estudiantes por cada uno de los cuadrantes dominantes del modelo $4 \mathrm{Q}$ y 2 estudiantes por cada una de las caracterizaciones del modelo de talento académico. De esta forma, se buscaba organizar por lo menos dos grupos que obedecieran al modelo $4 \mathrm{Q}$ y dos grupos que obedecieran al modelo de talento académico.

Igualmente, se pretendió que los estudiantes restantes, es decir, aquellos que no se podían ubicar claramente, se organizaran de manera aleatoria en grupos de trabajo. La Tabla 1 muestra la forma como se organizaron los grupos en cada curso. Respecto a la dinámica propia de cada grupo, la idea era que los integrantes se complementaran y se ayudaran entre sí, de forma que se pudiera mejorar el aprendizaje de cada uno a la luz de los logros propuestos para el grupo.

Tabla 1. Organización de grupos de trabajo

\begin{tabular}{ccccccccc}
\hline \multirow{2}{*}{ Año } & Sem & $\begin{array}{c}\text { Ests } \\
\text { 4Q }\end{array}$ & $\begin{array}{c}\text { Ests } \\
\text { T.A. }\end{array}$ & $\begin{array}{c}\text { Ests } \\
\text { Aleat }\end{array}$ & $\begin{array}{c}\text { Grps } \\
\mathbf{4 Q}\end{array}$ & $\begin{array}{c}\text { Grps } \\
\text { T.A. }\end{array}$ & $\begin{array}{c}\text { Grps } \\
\text { Aleat }\end{array}$ & $\begin{array}{c}\text { Tot } \\
\text { Ests }\end{array}$ \\
2014 & I & 8 & 6 & 6 & $2(4)$ & $2(3)$ & $2(3)$ & 20 \\
& II & 8 & 6 & 6 & $2(4)$ & $2(3)$ & $2(4)$ & 22 \\
2015 & I & 8 & 6 & 5 & $2(4)$ & $2(3)$ & $1(5)$ & 19 \\
& II & 8 & 6 & 7 & $2(4)$ & $2(3)$ & $2(4,3)$ & 21 \\
2016 & I & 8 & 6 & 10 & $2(4)$ & $2(3)$ & $2(5)$ & 24 \\
& II & 8 & 6 & 8 & $2(4)$ & $2(3)$ & $2(4)$ & 22 \\
\hline
\end{tabular}

Fuente: Elaboración propia.

De acuerdo con la Tabla 1, en todos los cursos se detectó claramente al menos a dos grupos completos de estudiantes que se ajustaban al modelo $4 Q$, pues se consiguió que, en cada grupo, hubiera un estudiante de cada perfil. De la misma forma, se logró conformar en cada curso, dos grupos del modelo TA (Talento Académico). Se permitió que los mismos estudiantes de cada curso se organizaran aprovechando que no se conocían y 
eso hacía que su proceso de organización como grupos de trabajo fuera más aleatorio. La cifra que aparece entre paréntesis indica la cantidad de estudiantes que conformaba cada grupo y la que aparece fuera del paréntesis indica la cantidad de grupos conformados. Siempre se mantuvo la organización de estos grupos durante el semestre y a todos los estudiantes se les impartieron las mismas clases magistrales, con las mismas explicaciones. También se les colocaron los mismos talleres y los mismos exámenes parciales para resolver en grupo. Precisamente, de eso se trataba esta parte del proyecto de investigación. Conviene precisar que la asignatura contó con 3 evaluaciones parciales con un peso porcentual del $20 \%$ y se conformaban de un taller (10\%) y de una prueba escrita (10\%).

El examen final tenía un peso porcentual de $30 \%$ y se concedió un $10 \%$ de motivación equivalente a una nota 5.0 para todos los estudiantes. A lo largo de cada semestre se presentaron algunas deserciones que obedecieron a razones absolutamente externas al método utilizado: Ubicación en otra ciudad, transferencia de universidad, transferencia de programa, situaciones familiares apremiantes, cambios en la situación económica fueron algunas de las razones por las cuales unos pocos estudiantes tuvieron que desertar de la carrera. Por una afortunada coincidencia, todos los casos se presentaron con estudiantes que habían conformado grupos en forma aleatoria, lo cual permite suponer que la muestra no se desbalanceó.

\section{Resultados}

En la Tabla 2, se presenta el promedio de las notas parciales obtenidas a lo largo del semestre en cada curso y en cada grupo.

Tabla 2. Promedio de notas obtenidas

\begin{tabular}{lccccccc}
\hline \multirow{2}{*}{ Año } & Sem & \multicolumn{2}{c}{ Modelo 4Q } & \multicolumn{2}{c}{ Modelo T.A. } & \multicolumn{2}{c}{ Modelo Aleatorio } \\
& Irp 1 & Grp 2 & Grp 1 & Grp 2 & Grp 1 & Grp 2 \\
2014 & I & 4.0 & 4.1 & 4.4 & 4.5 & 4.2 & 4.1 \\
& II & 3.6 & 3.8 & 3.9 & 3.9 & 4.0 & 4.1 \\
2015 & I & 3.7 & 3.8 & 3.5 & 3.4 & 4.5 & 4.6 \\
& II & 4.1 & 4.3 & 4.3 & 4.1 & 4.2 & 4.2 \\
2016 & I & 4.3 & 4.5 & 4.1 & 4.0 & 3.9 & 3.7 \\
& II & 4.0 & 4.1 & 4.0 & 4.1 & 4.4 & 4.4 \\
\hline
\end{tabular}

Fuente: Elaboración propia.

Finalizando el semestre, se solicitó a los estudiantes que respondieran por escrito dos preguntas. La primera era abierta e indagaba por su satisfacción respecto a su participación en el grupo que le había correspondido (de allí se infería si la respuesta era favorable o no al proceso investigativo), se procuró así que el estudiante se expresara libremente y de manera anónima, indicando solamente si había estado en grupo 4Q, grupo TA o en un grupo aleatorio. La segunda pregunta era cerrada: "¿A través de la 
metodología adoptada aprendió a programar? (explique el porqué)". La Tabla 3A presenta los resultados obtenidos teniendo en cuenta los siguientes criterios:

- Respuestas Muy Favorables (RMF), aquellas que se concentraron más en las ventajas y cuya respuesta a la segunda pregunta fue afirmativa.

- Respuestas Favorables (RF), aquellas que destacaron las ventajas pero cuya respuesta a la segunda pregunta fue negativa.

- Respuestas Desfavorables (RDF), aquellas que destacaron más las desventajas y su respuesta a la segunda pregunta fue positiva.

- Respuestas Muy Desfavorables (RMDF), aquellas que destacaron más las desventajas y su respuesta a la segunda pregunta fue negativa.

Tabla 3A. Tabulación de respuesta $1^{\mathrm{a}}$ pregunta

\begin{tabular}{|c|c|c|c|c|c|c|c|}
\hline Año & Sem & Tipo & RMF & RF & RDF & RMDF & Tot \\
\hline & & $4 Q$ & 6 & 2 & 0 & 0 & \\
\hline & I & T.A. & 6 & 0 & 0 & 0 & 19 \\
\hline \multirow{6}{*}{2014} & & Aleat & 3 & 1 & 1 & 0 & \\
\hline & & $4 Q$ & 5 & 3 & 0 & 0 & \\
\hline & II & T.A. & 5 & 1 & 0 & 0 & 22 \\
\hline & & Aleat & 4 & 4 & 0 & 0 & \\
\hline & & $4 Q$ & 5 & 3 & 0 & 0 & \\
\hline & I & T.A. & 4 & 2 & 0 & 0 & 18 \\
\hline \multirow{6}{*}{2015} & & Aleat & 2 & 1 & 1 & 0 & \\
\hline & & $4 Q$ & 6 & 1 & 1 & 0 & \\
\hline & II & T.A. & 4 & 1 & 1 & 0 & 20 \\
\hline & & Aleat & 3 & 1 & 0 & 0 & \\
\hline & & $4 Q$ & 5 & 2 & 1 & 0 & \\
\hline & I & T.A. & 3 & 3 & 0 & 0 & 22 \\
\hline \multirow{5}{*}{2016} & & Aleat & 4 & 3 & 1 & 0 & \\
\hline & & $4 Q$ & 6 & 2 & 0 & 0 & \\
\hline & II & T.A. & 3 & 2 & 1 & 0 & 21 \\
\hline & & Aleat & 3 & 3 & 1 & 0 & \\
\hline & \multicolumn{2}{|c|}{ Total } & 77 & 34 & 8 & 0 & 121 \\
\hline
\end{tabular}

Fuente: Elaboración propia.

Debe recordarse que el total de estudiantes por cada curso de la tabla $3 \mathrm{~A}$ no coincide numéricamente con el total presentado en la Tabla 1, debido a que hubo algunas deserciones durante el semestre. En relación con la segunda pregunta, la Tabla 3B presenta los resultados obtenidos según la breve encuesta que se hizo al finalizar el semestre a cada uno de los estudiantes. 
Tabla 3 B. Tabulación de respuestas $2^{\mathrm{a}}$ pregunta

\begin{tabular}{|c|c|c|c|c|c|c|}
\hline Año & Sem & Tipo & Sí & No & $\begin{array}{c}\text { No } \\
\text { Sabe }\end{array}$ & Tot \\
\hline \multirow{7}{*}{2014} & & $4 Q$ & 7 & 1 & 0 & \\
\hline & I & T.A. & 5 & 1 & 0 & 19 \\
\hline & & Aleat & 5 & 0 & 0 & \\
\hline & & $4 Q$ & 6 & 1 & 1 & \\
\hline & II & T.A. & 6 & 0 & 0 & 22 \\
\hline & & Aleat & 5 & 2 & 1 & \\
\hline & & $4 Q$ & 6 & 2 & 0 & \\
\hline \multirow{4}{*}{2015} & I & T.A. & 5 & 1 & 0 & 18 \\
\hline & & Aleat & 2 & 1 & 1 & \\
\hline & & $4 Q$ & 7 & 1 & 0 & \\
\hline & II & T.A. & 6 & 0 & 0 & 20 \\
\hline \multirow{8}{*}{2016} & & Aleat & 4 & 1 & 1 & \\
\hline & & $4 Q$ & 8 & 0 & 0 & \\
\hline & I & T.A. & 5 & 1 & 0 & 22 \\
\hline & & Aleat & 5 & 2 & 1 & \\
\hline & & $4 Q$ & 5 & 3 & 0 & \\
\hline & II & T.A. & 6 & 0 & 0 & 21 \\
\hline & & Aleat & 5 & 1 & 1 & \\
\hline & Tota I & & 98 & 19 & 6 & 121 \\
\hline
\end{tabular}

Fuente: Elaboración propia.

Se adoptó una respuesta "No Sabe" para aquellas respuestas que de manera clara y contundente no son un "Sí" ni un "No" absoluto.

\section{Discusión}

Si bien se puede recurrir a unas formas de selección y aplicación de modelos para caracterizar a los estudiantes que brinden un poco más de confiabilidad en su clasificación, también es cierto que este proceso implica más tiempo de análisis y que, por lo tanto, en las dos primeras semanas de interacción con los alumnos debe afinarse el proceso de observación y diálogo con ellos, así como la adopción de pruebas para detectar en dónde puede ubicarse con claridad hasta conformar los grupos necesarios. Se podría pensar que este proceso de investigación se realizaría mejor en un curso de II semestre; sin embargo en tal situación el concepto de conformación de grupos a la luz de la "aleatoriedad" se podría malograr dado que, después del $1^{\circ}$ semestre, los estudiantes ya se conocen y una conformación libre de grupos de trabajo podría no ser tan aleatoria como se desea. Tanto el modelo $4 \mathrm{Q}$ como el de talento académico y el aleatorio permiten obtener resultados significativos en el marco de este tipo de investigaciones en el aula. El modelo $4 \mathrm{Q}$ tiene todo el respaldo científico, aunque para la clasificación con alta confiabilidad debiera acudirse a un instrumento especializado cuyo costo y aplicación supera los alcances y recursos de este proyecto. Sin embargo, el estudio y apropiación del modelo $4 \mathrm{Q}$ permite tener unos elementos de juicio que, a partir de la interacción directa entre docente y estudiantes, puede lograr una clasificación con un alto porcentaje de efectividad siempre que se les cuente a los mismos estudiantes sus características y que 
ellos las validen en conjunto con el profesor.

El modelo de talento académico deriva, por su parte, de la experiencia de varios docentes investigadores y si bien parece ser simple, permite categorizar a los estudiantes en tres niveles fácilmente detectables, lo que le brinda una alta confiabilidad. En este modelo, con cada nivel de categorización, el docente tiene un objetivo específico dentro de su incorporación en los cursos, pero ello corresponde a la temática de otro artículo de investigación.

Por su parte, la conformación de grupos de manera aleatoria es la estrategia que normalmente se utiliza en los salones de clase por parte de los docentes de diferentes programas de formación profesional. Si bien, es el menos científico, se adoptó precisamente por ser el más utilizado y valía la pena confrontar sus resultados con lo que se obtuviera del análisis de otros modelos de conformación de grupos.

Dicho lo anterior, conviene decir que durante el proceso de investigación, y mientras los estudiantes realizaban sus talleres o sus pruebas escritas en grupo, se notaron los siguientes detalles en el desarrollo general del estudio:

- En los grupos 4Q, normalmente el que tomaba el liderazgo era el estudiante con perfil dominante social (Cuadrante $C$ ) $y$, en algunas ocasiones, se notaban discusiones sanas y cordiales discrepancias con el estudiante de cuadrante A (Lógico). La participación del estudiante con cuadrante dominante B (Secuencial) era mucho más operativa y el estudiante de cuadrante dominante D (Imaginativo) tenía la característica de participar mucho en la estructuración de las soluciones haciendo propuestas y, algunas veces, yendo mucho más allá de lo propuesto, aunque debe admitirse que siempre fue muy baja su participación en lo puramente operativo. Generalmente, la solución a los problemas y enunciados planteados venía de la mano del estudiante con cuadrante dominante Lógico.

- En los grupos de talento académico, se notó que el liderazgo siempre lo tomaba el estudiante AT (Altamente Talentoso). Aunque en algunas oportunidades, este se daba a la tarea de explicar sus soluciones, pocas veces abría espacios para el debate y la discusión. Los estudiantes BT (Bajamente Talentosos) participaban poco en el proceso aunque mostraban alta voluntad de realizar las tareas operativas. Los estudiantes MT (Medianamente Talentosos) siempre intentaban participar en la estructuración de las soluciones, trataban de discutir lo que correspondiera y, generalmente, adoptaban la tarea de ser los auditores del trabajo del estudiante BT, indicándole lo que debía hacer y cómo, en consonancia con las indicaciones del estudiante AT. Normalmente la solución a los problemas llega por el camino de las propuestas que hacía el estudiante tipo AT. 
- En los grupos aleatorios siempre existían por lo menos dos líderes y los debates y discusiones se daban entre ellos, mientras los demás esperaban pacientemente para que les indicaran qué debían hacer. Fue el tipo de grupo en el cual se realizaron mayores debates y discusiones. Las soluciones terminaban siendo concertadas a partir de dichos debates y al final todos participaban activamente.

\section{La Tabla 4 presenta un resumen de las características detectadas al interior de cada grupo a partir de su dinámica de interacción.}

\section{Tabla 4. Tabla de análisis de los grupos}

\begin{tabular}{|c|c|c|}
\hline $\begin{array}{l}\text { Tipo de } \\
\text { Grupo }\end{array}$ & Tópico & Análisis \\
\hline \multirow{10}{*}{$\begin{array}{l}\text { Grupo } \\
\text { basado } \\
\text { en el } \\
\text { Modelo } \\
4 Q\end{array}$} & Confiabilidad & $\begin{array}{l}\text { La clasificación de los estudiantes en cada uno de los cuadrantes que propone el } \\
\text { modelo implica un proceso de alta observación y de diálogo con cada uno. Se } \\
\text { requiere mucho tiempo e instrumentos para que esta clasificación sea } 100 \% \\
\text { confiable. El modelo es totalmente confiable e implica mucho estudio del mismo }\end{array}$ \\
\hline & Participación & $\begin{array}{l}\text { Los estudiantes participan en proporciones diferentes, pero de una u otra forma se } \\
\text { hacían oír }\end{array}$ \\
\hline & $\begin{array}{l}\text { Papel de cada } \\
\text { Estudiante }\end{array}$ & $\begin{array}{l}\text { El estudiante Lógico }(A) \text { junto con el Social }(C) \text { son los líderes. La parte operativa } \\
\text { la realiza el estudiante Secuencial }(B) \text { y el estudiante imaginativo (D) propone } \\
\text { permanentemente ideas de solución }\end{array}$ \\
\hline & $\begin{array}{l}\text { Discusiones y } \\
\text { Debates }\end{array}$ & $\begin{array}{l}\text { Las discusiones son breves, no se extienden mucho y normalmente se le concede } \\
\text { la razón al estudiante Lógico }(A)\end{array}$ \\
\hline & $\begin{array}{l}\text { Planteamiento } \\
\text { de Soluciones }\end{array}$ & $\begin{array}{l}\text { Normalmente se adoptan las soluciones que plantea el estudiante Lógico (A), dado } \\
\text { que sus argumentos normalmente son muy claros }\end{array}$ \\
\hline & $\begin{array}{l}\text { Trabajo } \\
\text { Operativo }\end{array}$ & $\begin{array}{l}\text { La parte operativa la realiza el estudiante Secuencial (B), nadie riñe con él por } \\
\text { esta labor. Este estudiante es el que menos participa en las discusiones }\end{array}$ \\
\hline & Concertación & $\begin{array}{l}\text { La concertación siempre se da alrededor de la solución que propone el estudiante } \\
\text { Lógico }(A)\end{array}$ \\
\hline & Interacción & $\begin{array}{l}\text { Fuera del grupo de trabajo interactúan muy poco entre sí, se limitan a conversar } \\
\text { durante la sesión de trabajo }\end{array}$ \\
\hline & Confiabilidad & $\begin{array}{l}\text { El modelo es bastante claro y es fácil de detectar a los estudiantes para ubicarlos } \\
\text { en cada una de las categorías }\end{array}$ \\
\hline & Participación & $\begin{array}{l}\text { Los estudiantes participan de manera activa pero se nota mucho más liderazgo del } \\
\text { estudiante AT. Los demás aceptan sus propuestas de solución }\end{array}$ \\
\hline \multirow{7}{*}{$\begin{array}{l}\text { Grupo } \\
\text { basado } \\
\text { en el } \\
\text { Modelo } \\
\text { Talento } \\
\text { Académico }\end{array}$} & $\begin{array}{l}\text { Papel de cada } \\
\text { Estudiante }\end{array}$ & $\begin{array}{l}\text { El estudiante AT propone soluciones, el estudiante BT realiza la parte puramente } \\
\text { operativa y el estudiante MT, a partir de dudas y preguntas, dinamiza la solución } \\
\text { que se adopta finalmente }\end{array}$ \\
\hline & $\begin{array}{l}\text { Discusiones y } \\
\text { Debates }\end{array}$ & $\begin{array}{l}\text { Hay muy poca discusión, los estudiantes MT y BT se ajustan a lo que propone el } \\
\text { estudiante AT. Hay bastantes preguntas por parte del estudiante MT }\end{array}$ \\
\hline & $\begin{array}{l}\text { Planteamiento } \\
\text { de Soluciones }\end{array}$ & $\begin{array}{l}\text { La solución siempre la plantea el estudiante AT y los demás miembros del grupo la } \\
\text { aceptan }\end{array}$ \\
\hline & $\begin{array}{l}\text { Trabajo } \\
\text { Operativo }\end{array}$ & $\begin{array}{l}\text { Toda la parte operativa es realizada por el estudiante BT con la supervisión del } \\
\text { estudiante MT }\end{array}$ \\
\hline & Concertación & $\begin{array}{l}\text { No hay concertación como producto de un debate, solo aceptan las soluciones que } \\
\text { plantea el estudiante AT }\end{array}$ \\
\hline & Interacción & Por fuera del grupo, tienen una interacción moderada \\
\hline & Confiabilidad & $\begin{array}{l}\text { El modelo es absolutamente aleatorio, es el más sencillo pues no requiere ningún } \\
\text { tipo de estudio, preparación o experiencia para conformar los grupos }\end{array}$ \\
\hline \multirow{5}{*}{$\begin{array}{l}\text { Grupo } \\
\text { basado } \\
\text { en el } \\
\text { Modelo } \\
\text { Aleatorio }\end{array}$} & Participación & $\begin{array}{l}\text { Los estudiantes intentan participar en la misma proporción, se siente todos al } \\
\text { mismo nivel, aunque se detecta que hay algunos estudiantes más talentosos que } \\
\text { otros }\end{array}$ \\
\hline & $\begin{array}{l}\text { Papel de cada } \\
\text { Estudiante }\end{array}$ & $\begin{array}{l}\text { Se distribuyen tareas y todos intentan cumplirlas con la mayor voluntad. Entre } \\
\text { ellos se dan a la tarea de supervisar lo que hacen los otros }\end{array}$ \\
\hline & $\begin{array}{l}\text { Discusiones y } \\
\text { Debates }\end{array}$ & $\begin{array}{l}\text { Hay mucha discusión y mucho debate, se plantean diferentes soluciones, se } \\
\text { discuten y se presentan con buenos argumentos }\end{array}$ \\
\hline & $\begin{array}{l}\text { Planteamiento } \\
\text { de Soluciones }\end{array}$ & $\begin{array}{l}\text { La solución se escoge a partir de la concertación que se deriva de los argumentos } \\
\text { que cada uno presente }\end{array}$ \\
\hline & Trabajo & El trabajo operativo es asignado también aleatoriamente. Todo el que recibe esta \\
\hline
\end{tabular}




\begin{tabular}{ll}
\hline Operativo & designación lo hace de buen agrado \\
Concertación & $\begin{array}{l}\text { Siempre existe concertación en la solución a adoptar frente a un enunciado o un } \\
\text { problema planteado } \\
\text { Por fuera del grupo tienen una alta interacción, parecieran proyectar la imagen de } \\
\text { Interacción }\end{array}$ \\
\hline
\end{tabular}

Fuente: Elaboración propia.

Las notas que se presentan en la Tabla 2 parecieran indicar que el trabajo en grupo es acertado tanto cuando obedece a un modelo preestablecido como cuando obedece a un criterio totalmente aleatorio. Sin embargo, debe admitirse que las observaciones de la Tabla 4 permiten tener un panorama mucho más aproximado de las características de interacción de cada uno de los tipos de grupos conformados. Las respuestas a la $1^{a}$ pregunta realizada al final del semestre (que se muestran tabuladas en la tabla $3 \mathrm{~A}$ ) son abrumadoramente positivas, pues las respuestas tipo RMF (Muy Favorables) y RF (Favorables) suman un $94 \%$ de efectividad en el proceso, dejando entrever que para los alumnos el trabajo en grupo investigativo ha sido exitoso.

Llama la atención que no hay respuestas tipo RMDF (Muy Des Favorables) lo cual deja solo un margen del 6\% de desfavorabilidad en el proceso que, para efectos estadísticos, es una cifra notoriamente baja. Al cuestionar las razones que llevaron a estas respuestas, se cuentan razones de índole personal, de gusto, razones según las cuales algunos estudiantes no querían trabajar en grupo y otras que se salen del ámbito académico. En relación con los datos tabulados en la tabla 3B, ante la pregunta "¿Aprendió a programar?", la respuesta también es abrumadoramente positiva a favor del "Sí", lo cual devela, ante todo, una satisfacción por parte de los estudiantes en lo que se refiere a su participación en esta investigación y en lo que han obtenido para sus propios logros de aprendizaje. El $80 \%$ de las respuestas fueron positivas, un $15 \%$ respondió negativamente y tan solo un $5 \%$ manifestó no tener elementos de juicio para saber si había aprendido a programar. El resultado de la investigación fue favorable en relación con los objetivos del curso y, según se presenta en la Tabla 4, se pueden hacer inferencias para aportar a la discusión acerca de la conformación de grupos de trabajo al interior del aula de clases en cursos de programación, allende del tradicional método aleatorio.

\section{Conclusión}

La investigación en el aula cada vez se hace más necesaria, especialmente para los cursos que son conducidos por docentes cuya formación en pregrado fue en áreas de la ingeniería. La adopción de diferentes modelos para la conformación de grupos y la comparación entre sus resultados es un insumo que bien puede servir de guía para realizar las actividades de los grupos de trabajo, la estrategia a seguir, o bien puede servir para que, luego de un análisis de los resultados, se posean más elementos de juicio para enriquecer las discusiones que al respecto se realicen en las salas de profesores. De la misma manera, vale destacar que aun cuando el modelo aleatorio no corresponde a un 
modelo con base científica, llama la atención que es el modelo que posibilita mayor cohesión entre los estudiantes y que, fuera del salón de clases, pareciera ser el que permite que el estudiante desarrolle con mayor efectividad la competencia de saber participar, interactuar, opinar, disentir, discutir, debatir y argumentar cuando se trate de grupos de trabajo, competencia que se considera de alta importancia en el marco de su futura vida profesional.

Cómo se destacó el paso de la modernidad a la posmodernidad, ha traído cambios estructurales en todos los sentidos. En el trabajo docente aquel pasado glorioso por el significativo papel para poder superar la ignorancia, los fanatismos y los cacicazgos fue en parte gracias al compromiso, dedicación, empatía, perseverancia y formación de los docentes.

En el caso de la historia en México los rasgos de los maestros han estado en estrecha correspondencia con el desarrollo y progreso nacional, por ejemplo: "compromiso", "solidaridad", "empatía", "promoción de la equidad" y "justicia social", aunadas a la "preparación y formación académica" han sido parte del involucramiento al trabajo social; sin embargo, en los últimos años mediante la puesta en marcha de políticas neoliberales se han reducido los roles sociales de los maestros y de manera paradójica se ha multiplicado lo administrativo y la priorización de: "calidad," "eficacia," y "eficiencia."

Con base a éste análisis se puede concluir, que de manera impositiva y no consensuada a través del tiempo se han ido transformando los rasgos de los maestros de manera significativa su campo de acción se ha reducido y se han impuesto nuevas normatividades; contradictoriamente, en lugar de preocuparse y dedicarse más porque sus alumnos aprendan tienden a ocuparse por recopilación de evidencias y aprobación de las evaluaciones.

Esta realidad tiende a favorecer la incertidumbre, dado que si no logran aprobar los exámenes de ingreso y permanencia serán considerados no idóneos y no podrán poder ejercer la docencia. Así pues, dicho análisis histórico permitió sustentar que los rasgos de los maestros del siglo XXI van mucho más allá de la aprobación de una evaluación, será necesario conocer y revalorar el pasado y así obtener elementos que permitan comprender e interpretar la pertinencia de los valores y su compromiso en el modelo de futuro docente quien se desarrolla entre la historia, competitividad, y la incertidumbre. 


\section{Referencias}

Adams, A. (2013). Cooperative learning effects on the classroom. (Tesis de maestría, Northern Michigan University). Recuperado de https://www.nmu.edu/education/sites/DrupalEducation/files/UserFiles/Adams_Anth ony_MP.pdf

Attard, A., Di Ioio, E., \& Geven, K. (2010). Student Centered Learning. An insight into theory and practice. Recuperado de http://www.ehea.info/media.ehea.info/file/T4SCL_forum_Leuven_October_2010/34 /2/2010_T4SCL_Stakeholders_Forum_Leuven__An_Insight_Into_Theory_And_Practice_600342.pdf

Ausubel, D. (1986). Sicología Educativa: Un punto de vista cognoscitivo. Ciudad de México: Trillas.

Barriga, A. F., \& Hernandez-Rojas, G. (2002). Estrategias docentes para un aprendizaje significativo: una interpretación constructivista. México: McGraw Hill Interamericana.

Blanchard, B. (2000). Ingeniería de Sistemas. Madrid: Isdefe.

Bruner, J. (2009). Actos de significado: Más allá de la revolución cognitiva. Boston, MA: Alianza Editorial SA.

Dooly, M. (2008). Constructing knowledge together. En Dooly, M. (Coord.), Telecollaborative Language Learning. A guidebook to moderating intercultural collaboration online (pp. 21-45). Bern: Peter Lang.

Herrmann, W. (2000). The whole brain. New York: McGraw Hill.

Kapp, K. (2012). Game based methods and estrategies for training and education. New York: Pfeiffer.

Lumsdaine, E., \& Lumsdaine, M. (2005). Creative Solving Problem: Thinking skills for a changing world. New York: McGraw Hill.

Piaget, J. (2001). Sicología y Pedagogía. México: Edtorial Crítica.

Prince, M. (2004). Does Active Learning work? Journal Engineering Education, 93(3), 131-153. 
Terenzini, P., Cabrera, A., Colbeck, C., Parente, J., \& Bjorklund, S. (2001). Collaborative Learning vs. Lectura / Discussion: Students reporting learning gains. Journal of Engineering Education, 93(3) 123-130.

Trejos-Buriticá, O. (2013). Significado y competencias. Pereira: Papiro.

Trejos, O. (2012). Aprendizaje en Ingeniería: un problema de comunicación. (Tesis Doctoral), Universidad Tecnológica de Pereira, Pereira, Colombia.

Van Roy, P. (2008). Techniques and methods in programming computer. Louvaine: University Press. 